\title{
Polyaniline Nanoparticles for the Selective Recognition of Aldrin: Synthesis, Characterization and Adsorption Properties
}

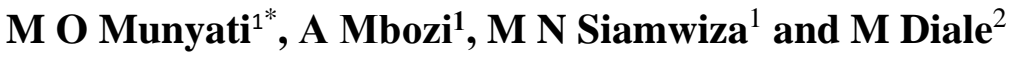 \\ ${ }^{1}$ Department of Chemistry, School of Natural Sciences, University of Zambia, P.O. Box \\ 32379, Lusaka, 10101 Zambia.2 \\ ${ }^{2}$ Department of Physics, Faculty of Natural \& Agricultural Sciences, \\ University of Pretoria, Private Bag X20, Hatfield, 0028 South Africa.
}

\section{Research highlight}

- Conducting polyaniline nanoparticles were prepared via inverse emulsion polymerization using aldrin as template and aniline as a functional monomer.

- Spherical particles with a size range that increased from $60-100 \mathrm{~nm}$ for nonimprinted which increased to between $500 \mathrm{~nm}-1500 \mathrm{~nm}$ for the imprinted.

- Conducting of materials prepared were determined to be $4.149 \mathrm{~S} / \mathrm{cm}$ for nanoimprinted and $0.546 \mathrm{~S} / \mathrm{cm}$ for imprinted

- Re-binding experiments showed the maximum amount of bound and point of saturation to be $0.799 \mathrm{ng} / \mu \mathrm{L}$ and $3.58 \mathrm{ng} / \mu \mathrm{L}$

\begin{abstract}
We report on the preparation, characterization and property evaluation of molecularly imprinted polyaniline nanoparticles that can be used for selective recognition of aldrin. Molecularly imprinted polyaniline (MI-PANI) nanoparticles were prepared by inverted emulsion polymerization using aldrin as a template and aniline as a functional monomer. Materials prepared were characterized using UV-VIS, FTIR and NMR for structural elucidation. Comparison of spectra data from UV-VIS, FTIR and NMR confirmed that aldrin was incorporated successfully into the polymer matrix. AFM and SEM were used to evaluate morphological characteristics. Both AFM and SEM revealed that the particles prepared were spherical in nature. The particle size was in range $60-100 \mathrm{~nm}$ for non-imprinted which increased to between $500-1500 \mathrm{~nm}$ for the imprinted. The surface morphology was observed to change from smooth to rough on incorporating aldrin molecules. Electrical properties were evaluated using a four-point probe coupled to a source meter. Non-imprinted materials showed an electrical conductivity of $4.149 \mathrm{~S} / \mathrm{cm}$ that reduced to $0.546 \mathrm{~S} / \mathrm{cm}$ in MI-PANI. The values of $K_{D}$ and $B_{\max }$ were found to be $0.6 \mathrm{ng} / \mu \mathrm{L}$ and $0.799 \mathrm{ng} / \mu \mathrm{L}$ respectively. Adsorption characteristics of aldrin and DDT were investigated to ascertain the selectivity of the imprinted nanoparticles. The results showed the distribution co-efficient of for DDT and Aldrin to be $0.76 \mathrm{ng} / \mathrm{ng}$ and $1.31 \mu \mathrm{L} / \mathrm{ng}$ respectively. This is indicative that the imprinted nanoparticles had a stronger affinity for Aldrin than DDT.
\end{abstract}

\footnotetext{
1* Author to whom correspondence should be addressed: Email: omunyati@unza.zm. Tel: +260 211295436
} 


\section{Graphical abstract}

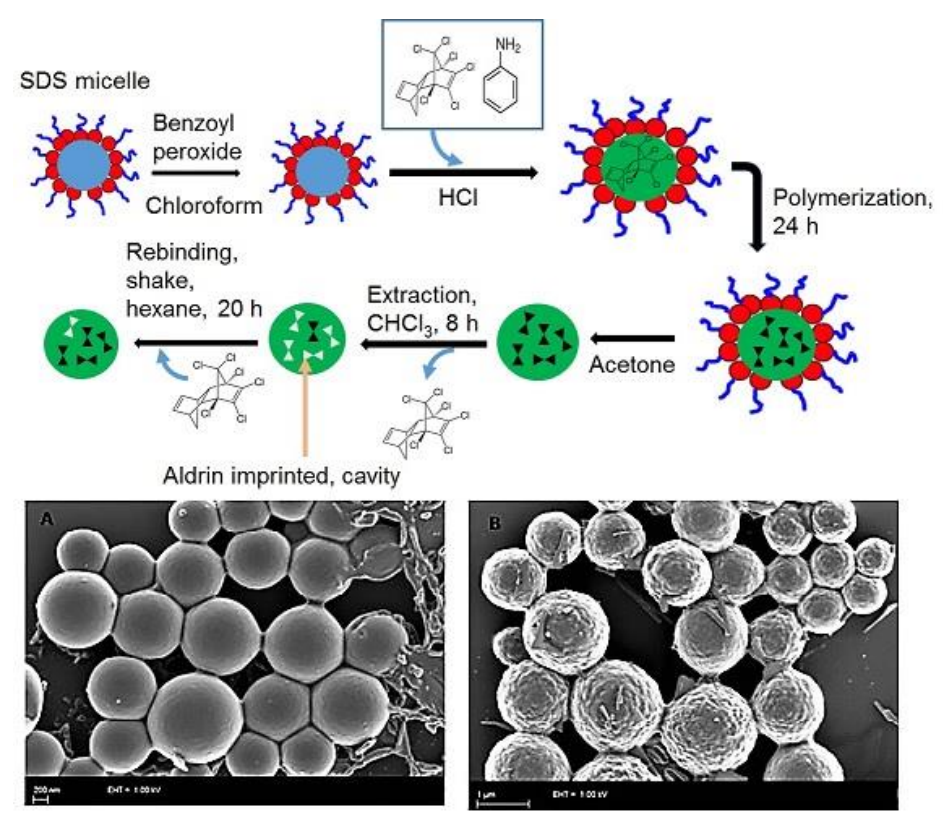

\section{Abbreviations}

AFM, Atomic force microscopy; APS, Ammonium persulfate; CP(s), Conducting polymer(s); FTIR, Fourier Transform Infra-red; HCl, Hydrochloric Acid; UV-VIS, Ultraviolet-visible spectroscopy; H-NMR, Proton-Nuclear Magnetic Resonance; I-V, Current -Voltage; KBr, Potassium Bromide; MIP, Molecularly Imprinted Polymer; MIP NP(s), Molecularly Imprinted Polymer Nanoparticle (s); MI-PANI, Molecularly Imprinted Polyaniline; MIT, Molecular imprinting technique; NPs, Nanoparticles; OCP(s) Organochlorine Pesticides(s); OLED, Organic light emitting diode; PANI, Polyaniline; S/cm, Siemens per centimetre; SDS, Sodium dodecyl sulphate; SEM, Scanning Electron Microscopy; TEM, Transmission Electron Microscopy; $\mathrm{K}_{\mathrm{D}}$, Equilibrium dissociation constant; C, Free equilibrium concentration; $\mathrm{B}_{\max }$, Apparent maximum binding capacity.

Keywords: molecularly imprinted polymers (MIPs), polyaniline, nanoparticles, aldrin, microemulsion, SEM, AFM, Langmuir model

\section{Introduction}

The reported accumulation of persistent organic pollutants (POPs)[1], in particular, organochlorine pesticides (OCPs) in the environment resulting from their use in the agriculture and public health sectors has continued to raise concern. These concerns have driven the development of low cost, easy to use devices that make use of novel smart materials with high specificity, selectivity, and sensitivities for analytes in sensing technologies. More specifically, there is a need for innovative methods for the synthesis of advanced "tailor-made" functional materials with specific recognition capacity and long-term performance stability. One of the 
most promising classes of new and highly selective functional materials are molecularly imprinted polymers (MIPs[2-6]. MIPs are one of the leading contenders in chemical sensor applications, combining selective recognition with chemical strength.

Conducting polymers are an important class of materials because of their potential applications in various electronic devices such as chemical sensors, electrochromic displays, and light emitting diodes[7]. Among the various conducting polymers, polyaniline (PANI) is one of the most promising conducting materials due to its facile synthesis, low cost, exceptional environmental stability, relatively high conductivity, and unique redox behavior. From these superiorities, PANI is used in applications such antistatic materials, anticorrosion coatings, batteries and energy storage, organic light-emitting diode (OLED), and chemical sensors. Although the use of conducting polymers (CPs) as transducers in chemical sensors is described in the literature, the associated sensing devices are limited due to lack of selectivity towards target analytes. With the evolution of molecular imprinting techniques (MIT), CPs can be chemically functionalized such that highly specific recognition sites are built within the polymer matrix resulting in an increase in selectivity[2-4]. Integration of molecularly imprinted conducting polymers as sensitive layers in chemical sensors provides a viable route for development of devices for selective and sensitive detection of environmental pollutants.

Molecularly imprinted polymers (MIPs) are commonly synthesized by bulk polymerization of monomers; this is done in the presence of templates, subsequently crushing or grinding, sieving and sedimentation of the polymer obtained. However, this process is tedious, time-consuming and the particles obtained show random shapes and size thus limiting their applicability. Also, the interaction sites created in the MIP are destroyed during grinding thereby reducing its loading and recognition capacity. Polymerisation strategies such as emulsion and precipitation polymerization are well-suited methods to obtain particles of defined shape and with the desired characteristics[8-10]. Emulsion polymerization, particularly, has gained attention due to better control of particle sizes and morphologies. MIP particles can be easily obtained using emulsion polymerization, the size can be controlled without difficulty and their integrity maintained. Also, the nano-MIPs prepared offer properties such as increased sensitivity due to the high surface area of the particles.

Typical preparation involves a matrix that is non-conductive. This makes the materials not ideal to function as a transducer. The use of conducting polymers provides an opportunity to develop a material that has specific recognitions but can also functions as a transducer. One of the most studied conducting polymer is polyaniline. This is due to its ease of synthesis, stability, 
good electrical and redox properties. Most MIP systems require addition of a crosslinker to maintain particle integrity, that is, to stabilize the imprinted binding sites and facilitate mechanical strength. The inclusion of non-conductive molecules in a conducting polymer matrix would be detrimental to its intrinsic electrical properties. In the case of polyaniline, its complex polymerization under certain conditions leads crosslinked 3-dimensional rigid structure. Polyaniline nanoparticles of defined shape and size can be prepared by emulsion polymerization[11-13]. When carried out in the presence of a template molecule, molecularly imprinted nanoparticle can be produced using a scalable process that avoids needing to grind to obtain desired particle size.

This paper describes a novel approach for the preparation and optimization of nanoparticles of molecularly imprinted polyaniline through inverted emulsion technique. Aldrin was used as a template molecule, and imprinted nanoparticles were evaluated for their optical, electrical and morphological characteristics. The capacity of the imprinted polyaniline nanoparticles to adsorb aldrin were evaluated through their adsorption properties using Langmuir model and Scatchard analysis while ability to selectively recognize Aldrin was done by comparing the adsorption characteristics of aldrin and DDT.

\section{Experimental}

\subsection{Reagent and chemicals}

Aniline, aldrin, sodium lauryl sulfate, benzoyl peroxide, hydrochloric acid, acetone, ethanol, and chloroform were all purchased from Sigma-Aldrich. All chemicals and solvents used in this work were used as received except for aniline which was double distilled before use. DDT was obtained from the University of Pretoria. MilliQ double-distilled ultrapure water was used in all experiments.

\subsection{Instrumentation}

The FTIR spectra were recorded using KBr pellets on a Perkin Elmer Spectrum RXI FTIR spectrometer. The sample was analyzed in pellet form by mixing the sample with pre-dried $\mathrm{KBr}$ in 1:5 ratio. The sample was scanned over a range of $400 \mathrm{~cm}^{-1}-4000 \mathrm{~cm}^{-1}$. ${ }^{1} \mathrm{H}-\mathrm{NMR}$ studies were performed using a Bruker Avance $400 \mathrm{MHz}$ NMR. The sample was analyzed in liquid form using deuterated chloroform as the solvent. AFM experiments were performed using a Bruker Dimension Icon AFM in scanAsyst mode. The measurements were carried out on polyaniline thin films. SEM images were recorded with a Zeiss Ultra Plus scanning electron 
microscope. Conductivity measurements were done using a four point probe coupled to a source meter. Quantitative analysis of aldrin and DDT in solutions was performed by GCTOFMS.

\subsection{Preparation of the imprinted polymeric nanoparticles}

\subsubsection{Polymer synthesis procedure}

Nanoparticles were prepared using the inverted emulsion polymerization. In the preparation of imprinted PANI NPs, $60 \mathrm{ml}$ of $0.2 \mathrm{M}$ benzoyl peroxide in chloroform was prepared, and $1.44 \mathrm{~g}$ sodium lauryl sulfate in $50 \mathrm{ml}$ of water $(0.1 \mathrm{M})$ was added to it under constant stirring at room temperature to obtain a milky white emulsion. A mixture of $1 \mathrm{~g}$ (10.84 mmol) aniline monomers, $8 \mathrm{mg}(0.0219 \mathrm{mmol})$ aldrin template in chloroform was taken and sonicated for $10 \mathrm{~min}$ to ensure that all material was dissolved. This solution was then slowly added to the milky emulsion and stirred for $30 \mathrm{~min}$. Dropwise addition of the dopant, $90 \mathrm{ml}(1.5 \mathrm{M} \mathrm{HCl})$ followed over a period of $30 \mathrm{~min}$. During the progress of the reaction, the colorless emulsion turns green. The reaction was allowed to proceed for 24 hours with stirring. The organic phase was separated and repeatedly washed with deionized water to remove all surfactant. The viscous organic solution was then treated with excess acetone to break the emulsion and precipitating the imprinted PANI NPs. It was then filtered, washed with acetone to remove any unreacted aniline. The dark green powder obtained was dried under vacuum at room temperature.

Soxhlet extraction was used to extract the aldrin from the polyaniline nanoparticles. The imprinted polymer was repeatedly treated with the dichloromethane solvent to remove the aldrin molecules. The extraction was allowed to proceed for 8 hours until the aldrin was washed off the polymer. Non-imprinted reference nanoparticles synthesized under identical conditions without the template aldrin. The general scheme for the preparation of molecularly imprinted polyaniline nanoparticles is shown diagrammatically below. 


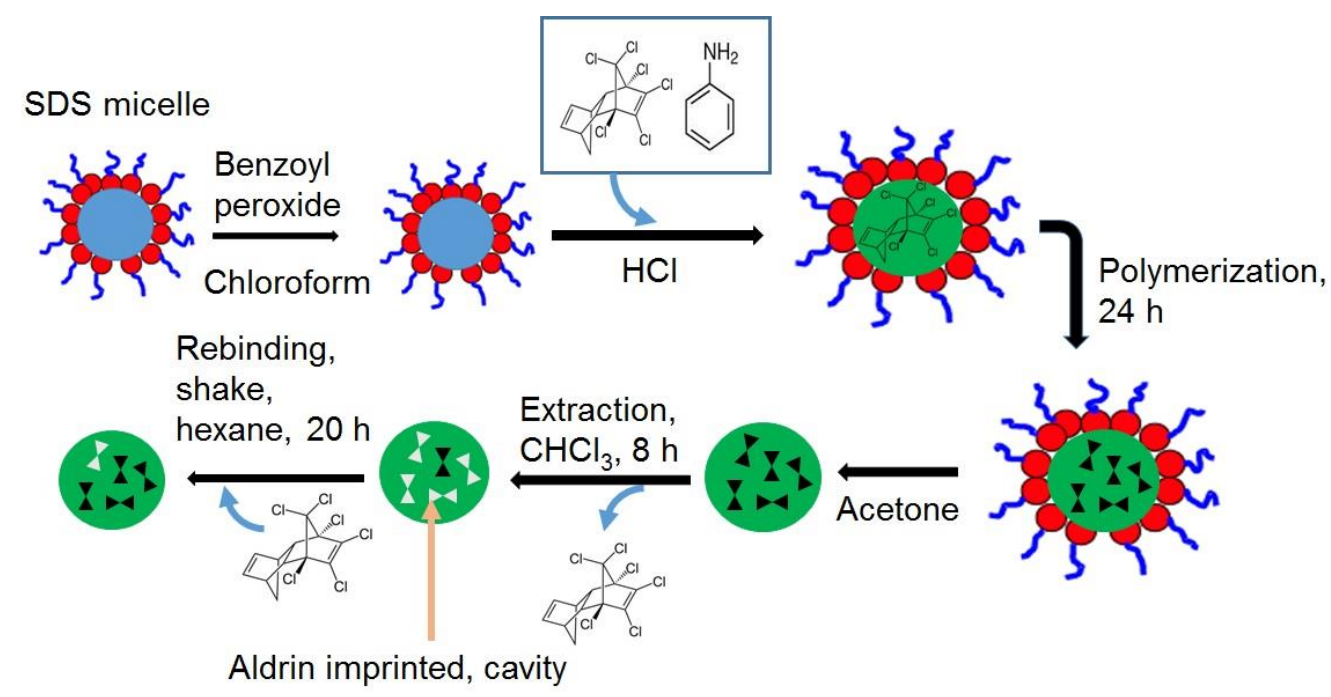

FIG. 1: Diagram showing the preparation of molecularly imprinted polyaniline nanoparticles

\subsubsection{Development of thin films}

The MI-PANI nanoparticles prepared were deposited on glass substrates. Before deposition, the glass substrates were washed with acetone, methanol and finally distilled water. After which they were placed in an oven and dried at $60{ }^{\circ} \mathrm{C}$. The method used for deposition was the dip coating method. The pre-cleaned glass substrate was dipped for 5 min into the imprinted or non-imprinted PANI dispersion of nanoparticles in chloroform. The slides were then withdrawn from the solution slowly to ensure the fluid had time to flow back down into the solution. The glass substrate was then held horizontally with the applied coating and left for 10 min until the solvent evaporated leaving a thin film.

\subsubsection{Rebinding experiments}

The binding capacity of the imprinted nanoparticles was determined using $40 \mathrm{mg}$ of MIPANI added into 5 test tubes with $1 \mathrm{ml}$ of aldrin at concentrations ranging from $1 \mathrm{ng} / \mu \mathrm{L}$ to 5 $\mathrm{ng} / \mu \mathrm{L}$ in hexane at room temperature for 20 hours with shaking. The polymer particles were then allowed to settle for $30 \mathrm{~min}$. The concentration of free aldrin in the solution was assayed by Gas Chromatography-Time of Flight Mass Spectrometry (GC-TOFMS). The amount of bound analyte to the molecularly imprinted polyaniline (MI-PANI) was calculated by subtracting the amount of free analyte from the initial concentration. The selectivity of the MIPANI was investigated by comparing the adsorption of aldrin and DDT on the MI-PANI. 


\section{Results and discussion}

\subsection{Structure elucidation}

The materials prepared were evaluated using FTIR and ${ }^{1} \mathrm{H}-\mathrm{NMR}$ for structural elucidation. Spectral analysis of both non-imprinted and imprinted materials was done to ascertain that the template molecule had been successfully incorporated into the nanoparticles. The molecular structure of polyaniline and the aldrin template are shown below.

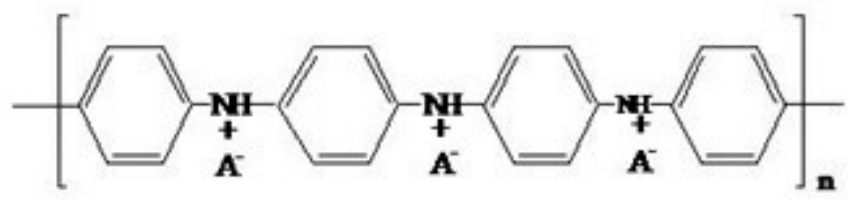

(1)

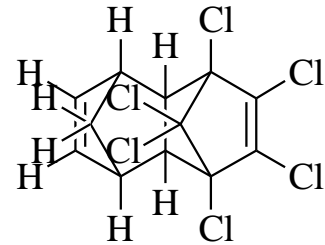

(2)

FIG. 2: Protonated emerald green polyaniline (1) and aldrin (2)

\subsubsection{Structure elucidation by Fourier transform infrared spectroscopy}

To confirm that polyaniline was synthesized according to plan, FTIR spectroscopic analysis was conducted to evaluate functional groups in the synthesized polyaniline (supplementary data). Fig. S1 shows the FTIR spectrum of PANI nanoparticles prepared at room temperature.

In the FTIR spectrum, the peak at $796 \mathrm{~cm}^{-1}$ is due to out of plane bending vibration of C$\mathrm{H}$ on para-disubstituted rings which is consistent with the proposed tetra-substituted benzene ring system $[14,15]$. The benzene ring connected at the para position with quinone diimine moieties and the out of plane bending vibration is evidenced by the presence of the absorption band at $830 \mathrm{~cm}^{-1}[16,17]$. The bands at $1570 \mathrm{~cm}^{-1}$ are due to the stretching vibration of $\mathrm{C}=\mathrm{C}$ quinoid ring[15,18]. The characteristic peak at $1480 \mathrm{~cm}^{-1}$ is due to the stretching vibration of $\mathrm{C}=\mathrm{C}$ of the benzenoid ring while and the bands at the $1294 \mathrm{~cm}^{-1}$ and $1236 \mathrm{~cm}^{-1}$ are ascribed to the $\mathrm{C}-\mathrm{N}$ stretching mode of the aromatic amine[14]. The strongest peak occurs at $1120 \mathrm{~cm}^{-1}$ which are assigned to the vibration mode of $-\mathrm{NH}^{+}=$and is indicative of a higher doping level[14,16]. Typically, a polymer that exhibits charge delocalization shows an absorption at around $1140 \mathrm{~cm}^{-1}$. The charge delocalization band for polyaniline $\mathrm{HCl}$ is reported to be at 1125 $\mathrm{cm}^{-1}[14,16]$. The absorption band at $3450 \mathrm{~cm}^{-1}$ is due to the N-H stretching mode. In general, anilinium ion displays a strong and broad band in the region $3400 \mathrm{~cm}^{-1}-3030 \mathrm{~cm}^{-1}$ due to N-H 
stretching vibration whereas N-H bending vibration appears at around $1302 \mathrm{~cm}^{-1}-1289 \mathrm{~cm}^{-}$ ${ }^{1}[16]$. Therefore, FTIR spectroscopic structural analysis confirmed that the nanoparticles prepared consisted of conductive polyaniline polymers[9,19-21].

FTIR characterization was performed to determine the functional groups in MIP before and after the extraction stage by using the $\mathrm{KBr}$ pellets. The spectra of the nanoparticles before removal of the template (a), after extraction of the template (b) and straight imprinted MIP, are shown in Fig. S2. The peaks are characteristic of the backbone structure of the different polymers synthesized. However, the absorption peak of the $\mathrm{N}-\mathrm{H}$ band at $3450 \mathrm{~cm}^{-1}$ is missing in spectra (b). It is suggested that this peak disappears due interactions between the template molecule (aldrin) and the polyaniline that hinder the N-H stretching modes which are restored after extraction of the template (a). The rest of the peaks at $1579 \mathrm{~cm}^{-1}, 1484 \mathrm{~cm}^{-1}, 1117 \mathrm{~cm}^{-1}$, $794 \mathrm{~cm}^{-1}$ are all in the polyaniline backbone as explained in the previous Sections.

\subsubsection{Structure elucidation by nuclear magnetic resonance spectroscopy}

${ }^{1} \mathrm{H}-\mathrm{NMR}$ was carried out on the non-imprinted and molecularly imprinted polymers. The spectra obtained is shown in Fig. S3 and Fig. S4. In Fig. S3, strong peaks in the region 7 ppm to $7.68 \mathrm{ppm}$ attributed to aromatic protons of the quinoid and benzenoid ring are present while the resonance at $3.66 \mathrm{ppm}$ is assigned to $\mathrm{N}-\mathrm{H}$ group protons. The peaks at $\delta 1.35 \mathrm{ppm}, 8.12$ ppm, and $8.13 \mathrm{ppm}$ may be due to the water protons bonded by $-\mathrm{NH}_{2}$ groups and $\mathrm{H}-\mathrm{N}^{+}$. The presence the peaks highlighted is consistent with polyaniline structure shown in the above Section[12,22].

The spectra of polyaniline nanoparticles imprinted with aldrin is shown in Fig. S4. Additional peaks arising from the presence of aldrin can be identified with the structure below.

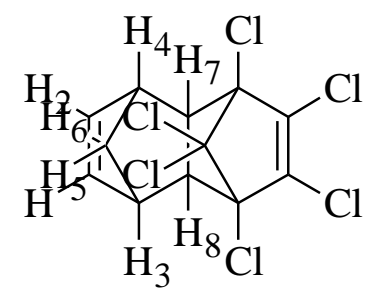

FIG. 3: Structure of aldrin with numbered protons

The resonance peaks at $\delta 6 \mathrm{ppm}$ in the form of a multiplet are associated with the protons 1 and 2[23]. Similarly, the peaks due to 7 and 8 protons are multiplets because of spin-spin splitting with a center at 2.7. The $\delta 2.2$ signals are assigned to protons 3 and 4 . The peaks at $\delta 2.7,2.9$ 
can be attributed to proton 3 and 4 in the aldrin structure. The peak at $5.17 \mathrm{ppm}$ is due to the presence of proton 1 and 2. Shifts in position are due to chemical interactions which may have taken place during the reaction. Fig. S4 shows the appearance of a peak at around $5.17 \mathrm{ppm}$ and 1. Finally, the methylene protons 5 and 6 which are anti-bridge and syn-bridge to other give peaks at $\delta 1.5$. Other peaks are recorded at $\delta 1.56,1.58,1.60 \mathrm{ppm}$ which can be attributed to the methylene protons in aldrin. Peaks at $5.6 \mathrm{ppm}, 1.58 \mathrm{ppm}, 1.6 \mathrm{ppm}$ in the spectrum of the aldrin MIP are assigned to the protons in aldrin moiety. These peaks are absent in the spectrum of the neat PANI, clear evidence that the modification was successful.

\subsubsection{Electronic spectra}

A typical UV-VIS spectra of the polyaniline base shows two distinct absorption bands between 315-345 and 610-650 $\mathrm{nm}$ according to Stejskal et.al. [24]. The position of absorptions with these bands depend on the structures formed which are influenced by the synthetics route used. Doping with acids generally leads to the appearance of three bands at 325-360 nm 400$430 \mathrm{~nm}$ and $780-826 \mathrm{~nm}$ with the disappearance of the band at $610-650 \mathrm{~nm}$ [25]. The two peaks often combined into a flat or distorted peak [24,26].

The UV-VIS spectra of the nonprinted and aldrin imprinted polyaniline nanoparticles are shown in Fig. S7. The spectra for the nonimprinted polyaniline shows absorption peak at 350 $\mathrm{nm}$ and $430 \mathrm{~nm}$ are ascribed to the $\pi-\pi^{*}$ transitions in the benzoid rings and exciton absorption in the quinoid rings[26-30]. The two peaks often combined into a flat or distorted peak. The absorption band at $800 \mathrm{~nm}$ is taken as evidence of the presence of localized polarons/bipolarons[28]. Similar absorption peaks are observed in the imprinted polyaniline nanoparticles. However, the peaks at $350 \mathrm{~nm}$ and $430 \mathrm{~nm}$ are seen to have shifted to $360 \mathrm{~nm}$ and $460 \mathrm{~nm}$ respectively exhibiting a redshift. It is also observed that the absorption peak characterizing the localized polarons/bipolarons essentially disappears at $800 \mathrm{~nm}$, instead significant broadening occurs [24,26,28,30]. 


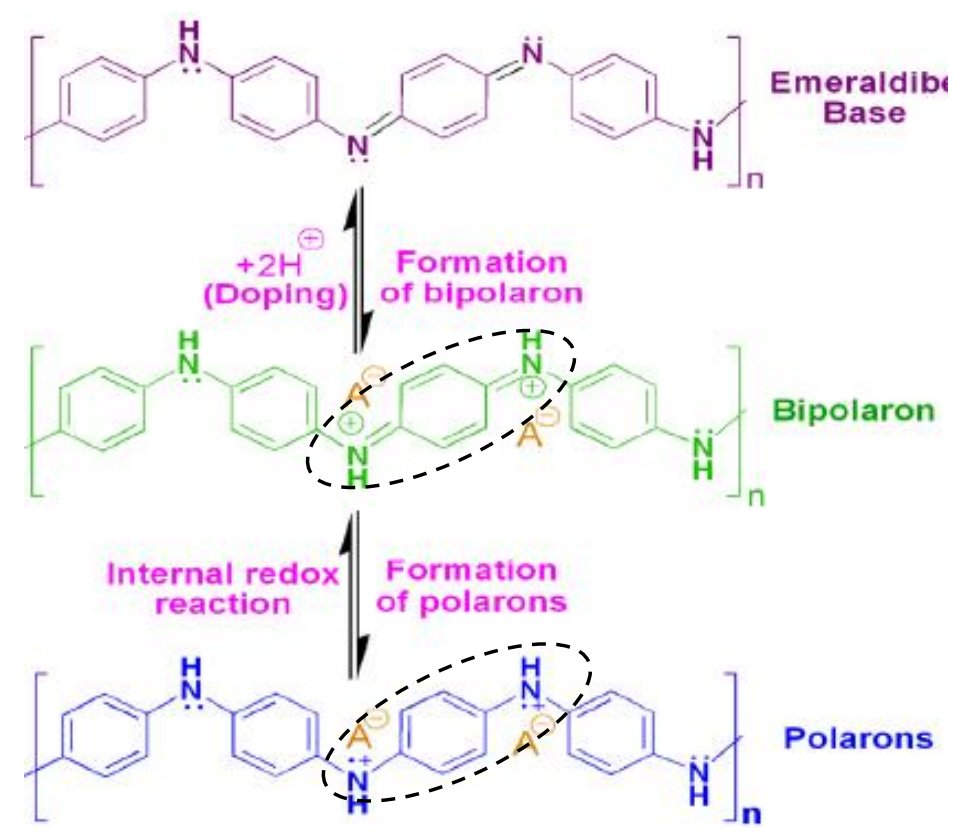

FIG. 4: Schematic diagram illustrating polyaniline protonic acid doping leading to the formation of polarons and bipolarons

The observed spectral changes can be attributed to arise from conformation changes arising from the interaction of the polyaniline chain and aldrin molecules. It has been suggested that the appearance of the carrier tail in the near infra-red is indicative of the delocalization of electron the polaron band. We hypothesized that the aldrin molecules act as "secondary dopants" which cause the structural changes of the polymer and consequently electronic transitions.

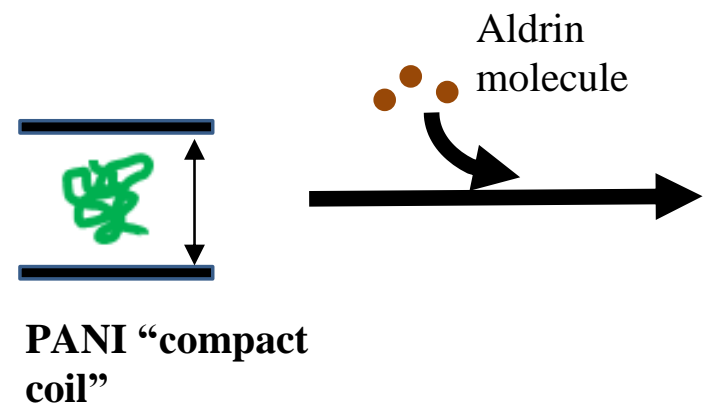

FIG. 5: Schematic diagram illustrating the induced conformation change arising from introduction of aldrin molecule.

A secondary dopant is an inert substance which when applied to polymer that has already doped (using a primary dopant) may induces further changes to the electronic, optical, magnetic and/or structural properties of the polymer[31,32]. An increase in conductivity may also 
accompany it. It is here postulated that the introduction of aldrin molecules led to a conformational change of the polyaniline chains from a more compact coil to more open (expanded) structure. The attainment of an expanded polymer backbone is accompanied by a reduction in $\pi$ conjugation defects that affects electronic spectra and transportation of charges. This invariably leads to more delocalization which is accompanied by a lowering of the band gap resulting in a slight shift of the $350 \mathrm{~nm}$ and $430 \mathrm{~nm}$ to longer wavelength.

\section{Morphological characteristics}

Physical properties of nanomaterials are highly influenced by their morphology and structural arrangement. Physical properties such as electrical conductivity are influenced by the ordering of both polymer chains and nanoparticles[33,34].

\subsection{SEM analysis of non-imprinted and imprinted polyaniline nanoparticles}

The scanning electron micrographs of the molecularly imprinted polyaniline and the nonimprinted polyaniline are shown in Fig. 6. Fig.6A revealed that the prepared nanoparticles were spherical in shape with particle size ranging from $60-100 \mathrm{~nm}$. The surface of the nonimprinted polyaniline was smooth. The imprinted particles were similarly spherically shaped with a surface that was rough compared to the non-imprinted (Fig. 6B). Furthermore, there was a general increase in size to an average of $500 \mathrm{~nm}-1800 \mathrm{~nm}$. The change in surface morphology may be due to near sub-surface imprinting. Particles are also observed to occur in clusters due to coalescence. The clustering can be attributed to agglomeration of primary particle agglomeration from a combination of Brownian motion and van der Waal attraction. Although Van der Waals force are weak forces, they become significant at very short distances (nanoscale). Particle interaction facilitated by Brownian motion ensures the nanoparticles colliding with each other all the time. The combination of van der Waals attraction force and Brownian motion would result in the formation of agglomeration of the nanoparticles. 

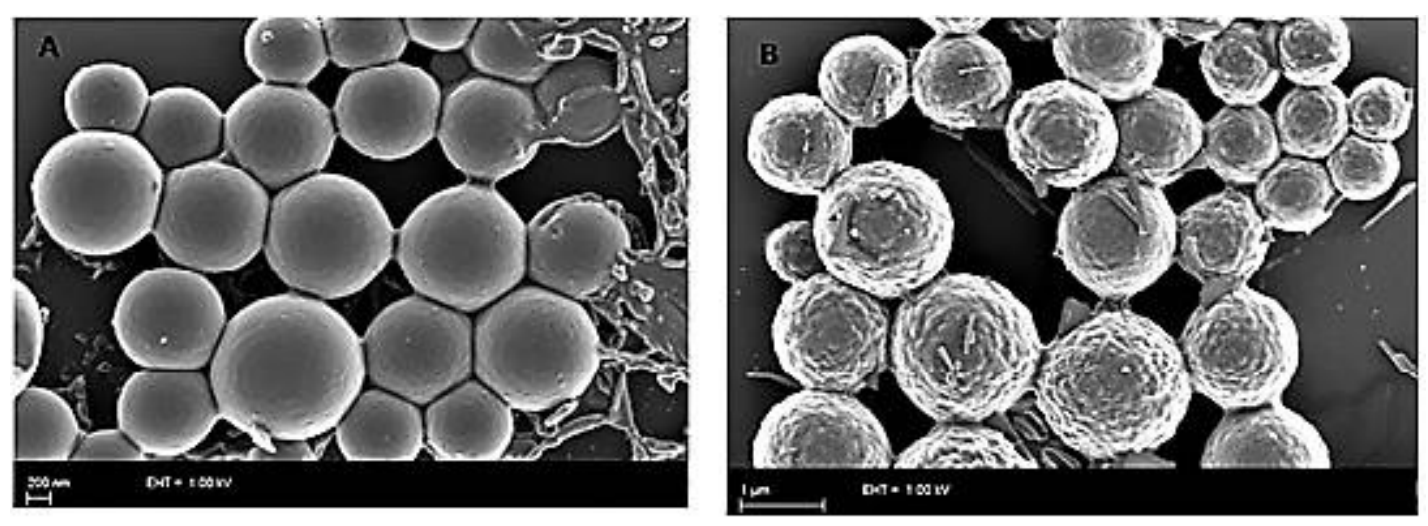

FIG. 6: Scanning electron micrographs: non-imprinted polyaniline: (A) nanoimprinted polyaniline (B) molecularly imprinted polyaniline

\subsection{AFM Studies of non-imprinted polyaniline and molecularly imprinted polyaniline}

AFM further confirmed particle morphology. Fig. 4 shows $5 \mu \mathrm{m}$ scanned AFM 3D height images for the neat PANI thin films, and MI-PANI carried out in contact mode. The images show particles that are spherically shaped. The AFM images further confirm that the nanoparticles are successfully synthesized according to plan. AFM studies were also carried out on molecularly imprinted polyaniline to study the surface morphology of the imprinted films. From Fig.4b, it can be noted that the imprinted nanoparticles were spherical in shape with a general increase in particle size. AFM results complement the SEM analysis.
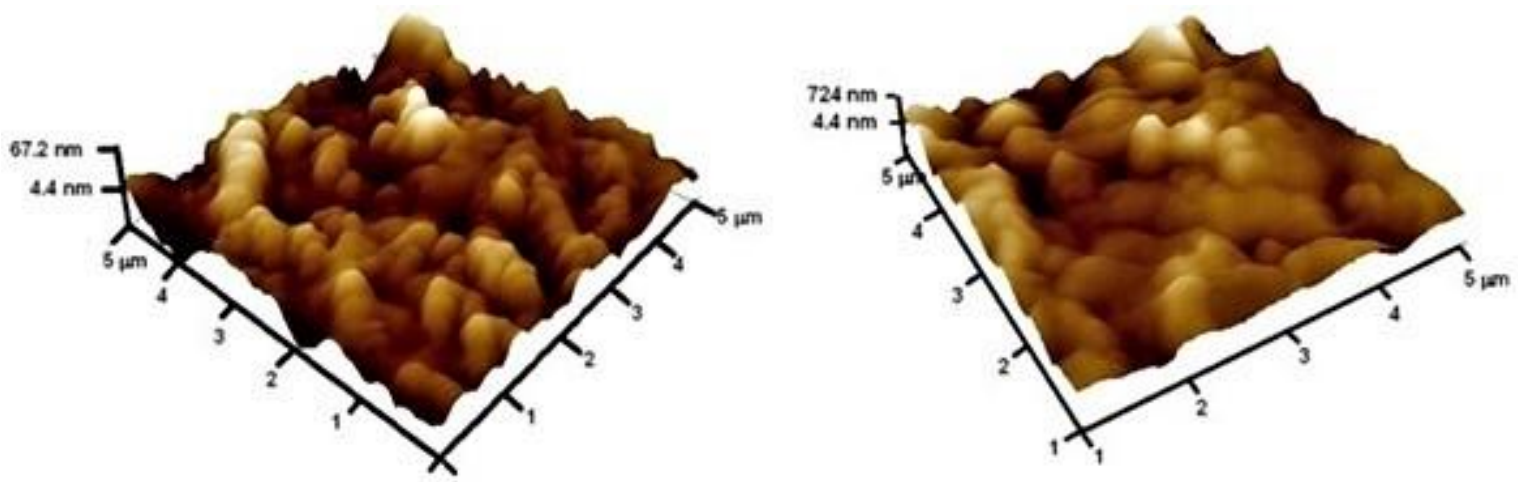

FIG. 7: AFM micrographs: (a) nanoimprinted polyaniline nanoparticles (b) molecularly imprinted polyaniline 


\section{Conductivity}

Current- voltage measurements were carried out at a start current and end current of $\quad 10$ $\mu \mathrm{A}$ and $50 \mu \mathrm{A}$ respectively. By use of four-point probe Labview analysis software, the voltage-current as well as resistivity-current curves were obtained and are shown in Fig. S6 and Fig. S7. The room temperature voltage-current characteristics of PANI and aldrin MI-PANI pellet are shown in Fig. S6. This curve indicates that the conductivity of PANI and MI PANI follow Ohmic behavior at room temperature. Fig. S7 shows the resistivity-current curves for the non-imprinted and imprinted materials. It is observed that the resistivity of polyaniline drastically increased after imprinting with aldrin.

The intrinsic electrical properties of conducting polymers arise from the conjugated molecular arrangement that enables the formation of delocalized electronic states. Recent studies have reported that charge transport in polyaniline nanoparticles occur primarily via interchain hopping. Furthermore, charge transport in conductive blends and percolated cluster has been reported as fractal-like [33]. This leads to the availability of random hopping site. The decrease in conductivity of polyaniline after imprinting with aldrin can be attributed to the disturbance of the inter-chain charge transport mechanism by having non-conductive species in the matrix.

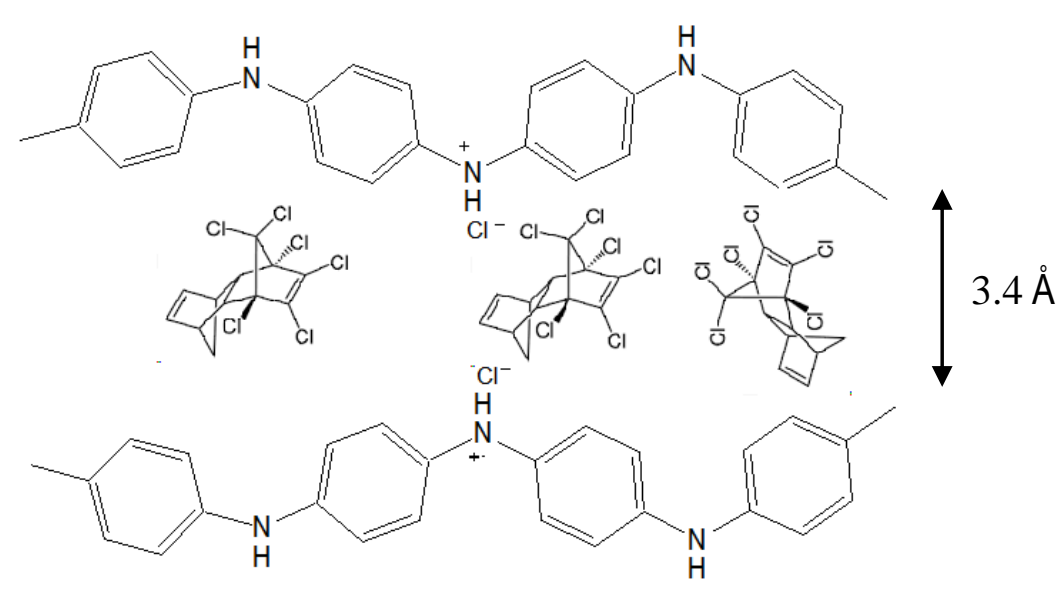

(a)

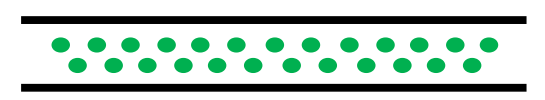

(b)

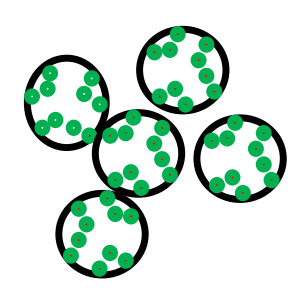

(c)

FIG. 8: Schematic representation showing: (a) the dopant counter ions and aldrin between the polyaniline chains, (b) Hoping sites within well aligned polymer chains inside a nanotube (c) Random hopping sites with a nanoparticle[33]. 
The conductive nature of polyaniline materials can be inferred from UV-VIS spectroscopic measurements (Fig. S7). The conformation changes that occur generally lead to increased conductivity which was not the case in this work as evidenced from the currentvoltage measurements. It is postulated that while the UV-VIS spectral data showed that incorporation of aldrin induced conformational changes that resulted in better conjugation. This would result in better intra-chain transport and hence an increase conductivity. Charge transport in polyaniline nanoparticles appears to be dominated by inter-chain hopping[33,35]. It has been suggested that interchain transport is relatively facile due to the presence of noninconsequential overlap between $\pi$ - orbitals of adjacent chains. Electron transfer is reported to via direct three-dimensional coupling or through intermediate dopant ions. The dopant ions can connect adjacent chains leading to the formation of polarons clusters resulting in a threedimensional network of polaron clusters. Presence of non-conducting aldrin molecules inbetween chains would affect the inter-chain conduction processes negatively as illustrated in Fig. 8 [27]. Hence, the conductivity reduced from $4.149 \mathrm{~S} / \mathrm{cm}$ to $0.546 \mathrm{~S} / \mathrm{cm}$ in the nonimprinted and imprinted respectively.

\section{Adsorption characteristics}

The binding properties of the imprinted polymers are evaluated through adsorption isotherms to establish the relationship between the concentration of bound and free guest in the system. Molecularly imprinted polymers (MI-PANI) were evaluated by rebinding experiments and the data fitted into the mathematical models the Langmuir isotherm [36-38].

\subsection{Equilibrium rebinding analysis}

The binding properties of MI-PANI were measured with initial concentrations of aldrin ranging from $1.4-5.0 \mathrm{ng} / \mu \mathrm{g}$ as shown in Table 1. Fig. S8 shows the binding profile of aldrin onto MI-PANI. The curve was obtained by plotting the saturated adsorbed amounts of aldrin against initial concentrations of the aldrin in hexane solution. In the concentration range investigated, the amount of aldrin adsorbed increases with the increasing aldrin initial concentration. This indicates that there are specific rebinding sites on the imprinted polymers. 
Table 1: Concentration $(\mathrm{ng} / \mu \mathrm{L})$ of aldrin in the sample solutions.

\begin{tabular}{cccc}
\hline Sample & $\begin{array}{c}\text { Initial Conc. } \\
\left(\mathrm{C}_{0}\right)\end{array}$ & $\begin{array}{c}\text { Con. at equilibrium } \\
\left(\mathrm{C}_{\mathrm{e}}\right)\end{array}$ & Bound aldrin \\
\hline 1 & 5 & 4.1 & 0.9 \\
2 & 4 & 3.4 & 0.6 \\
3 & 3 & 2.5 & 0.5 \\
4 & 2 & 1.8 & 0.2 \\
5 & 1.4 & 1.3 & 0.1 \\
\hline
\end{tabular}

\subsection{Langmuir isotherm}

The Langmuir isotherm assumes monolayer adsorption on a uniform surface with a limited number of adsorption sites. Once a site is in use, no further sorption can take place at that site. The surface will finally reach a saturation point where the maximum adsorption of the surface will be achieved. The data obtained in the batch binding experiment were fitted into the Langmuir model to obtain the plot in Fig. S9. The Langmuir equation is as shown below.

$$
B=\frac{B_{\max } \times C}{K_{D} \times C}
$$

where $K_{D}$ is the equilibrium dissociation constant, $\mathrm{C}$ is the free equilibrium concentration of aldrin, and $\mathrm{B}_{\max }$ is the apparent maximum number of aldrin molecules binding on the nanoparticles. From Fig. S9, it is observed that at equilibrium concentration higher than 3.58 $\mathrm{ng} / \mu \mathrm{L}$, adsorption of MIPs became stable as its recognition sites were almost saturated. Therefore, the apparent maximum amount of bound aldrin $\left(\mathrm{B}_{\max }\right)$ and the point of saturation are established to be $0.799 \mathrm{ng} / \mu \mathrm{L}$ and $3.58 \mathrm{ng} / \mu \mathrm{L}$ respectively.

\subsection{Scatchard analysis}

Scatchard analysis was employed to analyze further the binding isotherms, which is an approximate model commonly used to estimate the binding parameters of MIPs. The Scatchard equation can be expressed as follows:

$$
\frac{B}{C}=\frac{B_{\max }-B}{K_{D}}
$$

where $K_{D}$ is defined as the equilibrium dissociation constant of the binding sites, $B$ is the amount of aldrin bound to the polymer, $\mathrm{B}_{\max }$ is the maximum adsorption amounts of aldrin on 
the polymer, and $\mathrm{C}$ is the equilibrium concentration of aldrin in the solution. Fig. S10 shows the Scatchard plot of the binding of aldrin in MI- PANI.

The linear regression equation was, $\mathrm{B} / \mathrm{C}=-15.32 \mathrm{x}+13.28\left(\mathrm{R}^{2}=0.987\right)$, suggesting that the homogeneous recognition sites for aldrin were formed in the MI-PANI. The slope was established to be $-15.32=1 / \mathrm{K}_{\mathrm{D}}$, where $\mathrm{K}_{\mathrm{D}}$ is the equilibrium dissociation constant of the binding sites. The intercept, $13.28=\mathrm{B}_{\max } / \mathrm{K}_{\mathrm{D}}$, where $\mathrm{B}_{\max }$ is the maximum adsorption amounts of aldrin on the polymer. $\mathrm{K}_{\mathrm{D}}$ and $\mathrm{B}_{\max }$ were calculated to be $0.06 \mathrm{ng} / \mu \mathrm{L}$ and $0.799 \mathrm{ng} / \mu \mathrm{L}$ respectively.

\section{Molecular Selectivity of Polymers}

In order to verify that imprinted polymers are selective to aldrin, the binding of aldrin and a structurally related compound was investigated. DDT was chosen as the reference for investigating the specific selectivity.<smiles>ClC1=C(Cl)C2(Cl)CC(Cl)(C(Cl)=C2Cl)C1Cl</smiles>

(a)

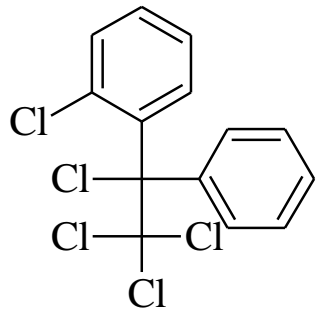

(b)

FIG. 9: Structural related molecules used for evaluation of the selectivity of imprinted nanoparticle: (a) aldrin (b) DDT

A higher binding affiity for aldrin was exhibited by the imprinted polymers compared to the structural related compound DDT. The distribution coefficient $K_{D}$ were utilized to evaluate the molecular selectivity of MI-PANI. $\mathrm{K}_{\mathrm{D}}$ is defined as follows:

$$
K_{D}=\frac{C_{p}}{C_{s}}
$$

where $C_{p}(n g / \mu L)$ is the amount of adsorbed aldrin on MI-PANI and $C_{s}(n g / \mu L)$ is the equilibrium concentration of substrates in solution. A linear function is the simplest and most widely used adsorption isotherm equation which is conventionally expressed in terms of the distribution coefficient $K_{D}$. 
Table 2: Comparison of distribution coefficient of Aldrin and DDT.

\begin{tabular}{|c|c|c|c|c||}
\hline Substrate & $\begin{array}{c}\text { Initial } \\
\text { concentration } \\
(\mathrm{ng} / \mu \mathrm{L})\end{array}$ & $\begin{array}{c}\text { Concentration at } \\
\text { equilibrium } \\
\mathrm{ng} / \mu \mathrm{L}\end{array}$ & $\begin{array}{c}\text { Bound } \\
(\mathrm{ng} / \mu \mathrm{L})\end{array}$ & $\begin{array}{c}\text { Distribution } \\
\text { coefficient K } \\
(\mu \mathrm{L} / \mathrm{ng})\end{array}$ \\
\hline Aldrin & 3 & 1.3 & 1.7 & 1.31 \\
\hline DDT & 3 & 1.7 & 1.3 & 0.76 \\
\hline
\end{tabular}

MIPs exhibited a high distribution coefficient of 1.31 for aldrin as compared to 0.76 for DDT. This indicates that higher recognition cavities were created in the resultant MIP nanoparticles. Furthermore, the high $\mathrm{K}_{\mathrm{D}}$ value obtained for aldrin suggested that the imprinting method created specific cavities based on the shape selection and position of functional groups that recognized template (aldrin). Therefore, MI- PANI exhibit much stronger affinity for aldrin than for DDT.

\section{Conclusion}

In the present study, nanoparticles of MI-PANI were prepared by inverted emulsion polymerization. Molecularly imprinted conducting polymers were produced using aniline as a functional monomer and aldrin as a template. FTIR and NMR were used for structural elucidation on the neat polymers and the imprinted. Morphological characterization was done by SEM and AFM which showed that the particles synthesized were spherical in shape. The non-imprinted MIPs had a smooth surface with diameters ranging from $60 \mathrm{~nm}-100 \mathrm{~nm}$. Molecular imprinting resulted in particles that were spherical, with rough surfaces and increased size in the range $500 \mathrm{~nm}-1500 \mathrm{~nm}$ due to incorporating aldrin molecules in the matrix. Neat PANI had a conductivity of $4.149 \mathrm{~S} / \mathrm{cm}$ which reduced to $0.546 \mathrm{~S} / \mathrm{cm}$. The drastic reduction in conductivity was suggestive that a significant amount of template-monomer had incorporated into the matrix. Results from this study demonstrate that polyaniline nanoparticles could successfully be imprinted with aldrin and can be subsequently removed leaving potential recognition sites. Adsorption properties evaluated through the Langmuir adsorption isotherm and Scatchard analysis. The apparent maximum amount of bound aldrin and the point of 
saturation was found to be $0.799 \mathrm{ng} / \mu \mathrm{L}$ and $3.58 \mathrm{ng} / \mu \mathrm{L}$ respectively. The specificity and selectivity of the imprinted polymers were investigated by binding analysis using aldrin and DDT. The results indicated that the imprinted polymers showed good selectivity and specificity for aldrin. Therefore, the synthesized imprinted polymers can potentially be used as active layers, for selective and sensitive detection of aldrin in chemical sensors.

\section{Appendix: Supplementary data}

Supplementary data associated with this article can be found in the online version at http://dx.doi.org.

\section{ACKNOWLEDGEMENTS}

This project was carried out with the financial support from the International Programme in Physical and Chemical Sciences, Uppsala University, Sweden. The authors wish to express sincere gratitude to the University of Pretoria for the use of their facilities to carry out the characterization of various materials and technical assistance.

\section{REFERENCES}

[1] L. Ritter, K.R. Solomon, J. Forget, M. Stemeroff, C. O’Leary, A Review of Selected Persistent Organic Pollutants, Apostila. (1995) 1-149.

[2] T. Khursheed, M.U. Islam, M. Asif Iqbal, I. Ali, A. Shakoor, M.S. Awan, A. Iftikhar, M. Azhar Khan, M. Naeem Ashiq, Synthesis and characterization of polyanilinehexaferrite composites, Journal of Magnetism and Magnetic Materials. 393 (2015) 814. doi:10.1016/j.jmmm.2015.05.008.

[3] G. Guan, B. Liu, Z. Wang, Z. Zhang, Imprinting of molecular recognition sites on nanostructures and its applications in chemosensors, Sensors. 8 (2008) 8291-8320. doi:10.3390/s8128291.

[4] M. Peeters, Molecularly Imprinted Polymers ( Mips ) for Bioanalytical Sensors : Strategies for Incorporation of Mips into Sensing Platforms, Austin Journal of Biosensors \& Bioelectronics. 1 (2015) 1-5.

[5] H. Yan, K.H. Row, Characteristic and Synthetic Approach of Molecularly Imprinted Polymer, International Journal of Molecular Sciences. 7 (2006) 155-178. doi:10.3390/i7050155.

[6] N.A. Alenazi, J.M. Manthorpe, E.P.C. Lai, Selectivity enhancement in molecularly imprinted polymers for binding of bisphenol A, Sensors (Switzerland). 16 (2016). doi:10.3390/s16101697.

[7] B. Scrosati, Conducting Polymers and Their Applications, Materials Science Forum. 42 (1989) 207-220. doi:10.4028/www.scientific.net/MSF.42.207. 
[8] S. Bhadra, D. Khastgir, N.K. Singha, J.H. Lee, Progress in preparation, processing and applications of polyaniline, Progress in Polymer Science (Oxford). 34 (2009) 783-810. doi:10.1016/j.progpolymsci.2009.04.003.

[9] J. Jang, J. Ha, S. Kim, Fabrication of polyaniline nanoparticles using microemulsion polymerization, Macromolecular Research. 15 (2007) 154-159.

doi:10.1007/BF03218767.

[10] P.S. Rao, S. Subrahmanya, D.N. Sathyanarayana, Inverse emulsion polymerization : a new route for the synthesis of conducting polyaniline, 128 (2002) 311-316.

[11] S. Palaniappan, A. John, Polyaniline materials by emulsion polymerization pathway, Progress in Polymer Science. 33 (2008) 732-758.

doi:10.1016/j.progpolymsci.2008.02.002.

[12] P.S. Rao, D.N. Sathyanarayana, S. Palaniappan, Polymerization of aniline in an organic peroxide system by the inverted emulsion process, Macromolecules. 35 (2002) 4988-4996. doi:10.1021/ma0114638.

[13] G. Dvorakova, R. Haschick, K. Chiad, M. Klapper, A. Biffis, Molecularly Imprinted Nanospheres by Nonaqueous Emulsion Polymerization, Macromolecular Rapid Communication. 32 (2010) 2035-2040. doi:10.1002/marc.201000406.

[14] E. C. Gomes, M. A. S. Oliveira, Chemical Polymerization of Aniline in Hydrochloric Acid $(\mathrm{HCl})$ and Formic Acid $(\mathrm{HCOOH})$ Media. Differences Between the Two Synthesized Polyanilines, American Journal of Polymer Science. 2 (2012) 5-13. doi:10.5923/j.ajps.20120202.02.

[15] A.C. Roy, V.S. Nisha, C. Dhand, M.A. Ali, B.D. Malhotra, Molecularly imprinted polyaniline-polyvinyl sulphonic acid composite based sensor for para-nitrophenol detection, Analytica Chimica Acta. 777 (2013) 63-71. doi:10.1016/j.aca.2013.03.014.

[16] W. Shao, R. Jamal, F. Xu, A. Ubul, T. Abdiryim, The effect of a small amount of water on the structure and electrochemical properties of solid-state synthesized polyaniline, Materials. 5 (2012) 1811-1825. doi:10.3390/ma5101811.

[17] I.H.Y.F.F. Ueda, Vibrational spectra and structure of polyaniline and related compounds, Syth.Met. E303 (1989).

[18] P. Mondal, K. Roy, S.P. Bayen, P. Chowdhury, Synthesis of polypyrrole nanoparticles and its grafting with silica gel for selective binding of chromium(VI)., Talanta. 83 (2011) 1482-6. doi:10.1016/j.talanta.2010.11.037.

[19] P.K.S. and V.K.S. I D Sharma, Structural, optical, morphological and electrical characteristics of polyaniline for device applications, 20 (2013) 145-149.

[20] N. Li, Y. Xiao, C. Xu, H. Li, X. Yang, Facile preparation of polyaniline nanoparticles via electrodeposition for supercapacitors, International Journal of Electrochemical Science. 8 (2013) 1181-1188. http://www.scopus.com/inward/record.url?eid=2-s2.084873816942\&partnerID=40\&md5=873673a4de890636df50cb609f4efe94.

[21] F. Zeng, Z. Qin, B. Liang, T. Li, N. Liu, M. Zhu, Polyaniline nanostructures tuning with oxidants in interfacial, Progress in Natural Science: Materials International. 25 (2015) 512-519. doi:10.1016/j.pnsc.2015.10.002. 
[22] R. Abdelkader, H. Amine, Mohammed, H-NMR spectra of conductive, anticorrosive and soluble polyaniline exchanged by an eco-catalyst layered ( Maghnite-H + ), Journal of Scientific Research Review. 2 (2013) 86-92.

[23] E.. Mooney, ed., Annual Reports on NMR Spectroscopy APL, Volume 4, Elsevier Science, 1971.

[24] J.S. and P. Kratochv, Polyaniline dispersions 2: UV-Vis Spectra, Synthetic Metals. (1993) 225-231. doi:10.1016/0379-6779(93)91266-5.

[25] T. Memorandum, A Literature Survey of Polyaniline , Part 1 Defence R \& D Canada, (2003).

[26] B.J. Kim, S.G. Oh, M.G. Han, S.S. Im, Synthesis and characterization of polyaniline nanoparticles in SDS micellar solutions, Synthetic Metals. 122 (2001) 297-304. doi:10.1016/S0379-6779(00)00304-0.

[27] B.-J. Kim, S.-G. Oh, M.-G. Han, S.-S. Im, Synthesis and characterization of polyaniline nanoparticles in SDS micellar solutions, Synthetic Metals. 122 (2001) 297-304. doi:10.1016/S0379-6779(00)00304-0.

[28] H. Wang, H. Wen, B. Hu, G. Fei, Y. Shen, L. Sun, D. Yang, Facile approach to fabricate waterborne polyaniline nanocomposites with environmental benignity and high physical properties, Nature Publishing Group. (2017) 1-12. doi:10.1038/srep43694.

[29] D. Jaaoh, C. Putson, N. Muensit, Enhanced strain response and energy harvesting capabilities of electrostrictive polyurethane composites filled with conducting polyaniline, Composites Science and Technology. 122 (2016) 97-103. doi:10.1016/j.compscitech.2015.11.020.

[30] V.J. Babu, S. Vempati, S. Ramakrishna, Conducting Polyaniline-Electrical Charge Transportation, Materials Sciences and Applications. 2013 (2013) 1-10.

[31] A.G.M. and A.J.E. Yong Gang Min, Younan Xia, Vapour phase "secondary doping" of polyaniline, Synthetic Metals. 69 (1995) 159-160.

[32] A.G. MacDiarmid, A.J. Epstein, Secondary doping in polyaniline, Synthetic Metals. 69 (1995) 85-92. doi:10.1016/0379-6779(94)02374-8.

[33] C. Nath, A. Kumar, Fractal like charge transport in polyaniline nanostructures, Physica B: Condensed Matter. 426 (2013) 94-102. doi:http://dx.doi.org/10.1016/j.physb.2013.06.019.

[34] H.D. Tran, J.M. D’Arcy, Y. Wang, P.J. Beltramo, V.A. Strong, R.B. Kaner, The oxidation of aniline to produce "polyaniline": a process yielding many different nanoscale structures, J. Mater. Chem. 21 (2011) 3534-3550. doi:10.1039/C0JM02699A.

[35] R.C. Hiorns, Review of electronic and optical properties of semiconducting $\pi$ conjugated polymers : applications in optoelectronics, 1412 (2004) 1397-1412. doi:10.1002/pi.1587.

[36] N.A. Yusof, S.K.A. Rahman, M.Z. Hussein, N.A. Ibrahim, Preparation and 
characterization of molecularly imprinted polymer as SPE sorbent for melamine isolation, Polymers. 5 (2013) 1215-1228. doi:10.3390/polym5041215.

[37] R.M. Rad, L. Omidi, H. Kakooei, F. Golbabaei, Adsorption of Polycyclic Aromatic Hydrocarbons on Activated Carbons : Kinetic and Isotherm Curve Modeling, International Journal of Occupational Hygiene. 6 (2014) 43-49.

http://ijoh.tums.ac.ir/index.php/ijoh/article/download/188/168.

[38] Z. Li, G. Yang, S. Liu, Y. Chen, Adsorption isotherms on nicotinamide-imprinted polymer stationary phase., Journal of Chromatographic Science. 43 (2005) 362-6. http://www.ncbi.nlm.nih.gov/pubmed/16176649. 